\title{
Early arteriosclerosis and its risk factors in subjects with prediabetes and new-onset diabetes
}

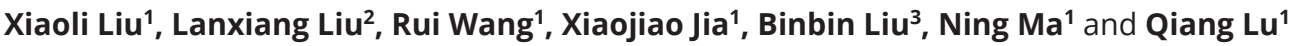 \\ 'Department of Endocrinology, The First Hospital of Qinhuangdao, Qinhuangdao, China \\ 2Department of Medical Imaging, The First Hospital of Qinhuangdao, Qinhuangdao, China \\ ${ }^{3}$ Department of Functional Examination, The First Hospital of Qinhuangdao, Qinhuangdao, China
}

Correspondence should be addressed to Q Lu: luqiang_tg@163.com

\begin{abstract}
Background: We aimed to investigate early arteriosclerosis and its risk factors in populations with prediabetes and new-onset diabetes.

Materials and methods: A total of 148 participants who did not have diabetes mellitus were assigned to three groups through an oral glucose tolerance test: the normal glucose tolerance (NGT) group; the impaired glucose regulation, also known as prediabetes group and the new-onset type 2 diabetes mellitus group. The insulin resistance index was assessed using the homeostatic model assessment of insulin resistance (HOMA-IR). An ELISA was used to determine the level of fibroblast growth factor 21 (FGF21). An arteriosclerosis detector was used to measure the brachial-ankle pulse wave velocity (baPWV) and ankle-brachial index (ABI). The baPWV, ABI, and FGF21 were used to assess early arteriosclerosis.

Results: Significant differences in age, systolic blood pressure (SBP), fasting plasma glucose (FPG), 2-h plasma glucose (2hPG), 2-h insulin (2hINS), and HOMA-IR were found between the NGT group and the prediabetes and new-onset diabetes groups. All of the above, except 2hINS, showed an increasing trend. Moreover, the FGF21 was higher in the new-onset diabetes group than in the NGT group. The baPWV was higher in the new-onset diabetes group than in the other two groups, but no significant difference was noted in the ABI. Age, SBP, diastolic blood pressure, FPG, 2hPG, and FGF21 were positively correlated with the baPWV. In addition, FPG, SBP, FGF21, and HOMA-IR were independent risk factors for the baPWV.

Conclusions: Patients with prediabetes and new-onset diabetes may have more significant early arteriosclerosis. The blood glucose level and insulin resistance index may be independent risk factors for early arteriosclerosis.
\end{abstract}

\author{
Key Words \\ - new-onset diabetes \\ - prediabetes \\ - early arteriosclerosis \\ - metabolism
}

Endocrine Connections (2021) 10, 599-606

\section{Background}

Many evidence-based medical studies have confirmed that cardiovascular and cerebrovascular events are the end-point events of patients with diabetes and that the pathological bases of diabetic macrovascular lesions are the structural and functional lesions of arteries characterized by arteriosclerosis. Studies have shown that the severity and location of arterial sclerosis are sensitive and specific indicators for predicting the risk of cardiovascular events, stroke, and amputation in patients with diabetes (1). The change in arterial stiffness occurs earlier than the https://ec.bioscientifica.com https://doi.org/10.1530/EC-21-0202 (c) 2021 The authors Published by Bioscientifica Ltd
This work is licensed under a Creative Commons Attribution-NonCommercial-NoDerivatives 4.0 Internationab ticense.ifica.com at 04/26/2023 01:23:13PM 
change in arterial structure. Early detection and active intervention can effectively eliminate or reduce the serious consequences of vascular events.

Decreased elasticity is a good, specific and sensitive indicator reflecting arterial vascular lesions in the early stage. The brachial-ankle pulse wave velocity (baPWV) is currently a classic indicator for assessing arterial vascular elasticity. It can effectively detect early changes in the compliance of large- and medium-sized arteries. It is currently recognized as the optimal method for measuring arterial stiffness and has the advantages of simplicity, noninvasiveness, reliability, and good repeatability (2). A higher baPWV value may suggest higher stiffness of the arterial blood vessels and decreased elasticity. The baPWV can quickly and accurately evaluate the stiffness of the arterial system and identify early vascular lesions, which is conducive to early intervention and control of vascular lesions $(3,4,5)$. The ankle-brachial index (ABI) can specifically and sensitively reflect the stenosis, obstruction, and calcification of the arterial blood vessels in the lower limbs caused by atherosclerosis. It is an accurate, reliable, and noninvasive detection index for the early diagnosis of peripheral arterial diseases $(6,7)$. Fibroblast growth factor 21 (FGF21) is a metabolic regulator that has multiple functions, such as reducing blood glucose and lipids, increasing insulin sensitivity, and reversing hepatic steatosis. Recent studies have found that increasing the concentration of FGF21 in the blood can delay vascular endothelial aging (8) and that FGF21 can be used as a cell biological marker of vascular aging.

It has been proven that a decrease in arterial elasticity is closely related to the level of blood glucose. Hyperglycemia can accelerate the occurrence of arteriosclerosis $(9,10)$. Even when diabetes has not yet occurred, an abnormal change in the blood glucose level may induce the occurrence of early arteriosclerosis and an increase in the blood glucose can further aggravate it. However, there have been few studies on early arteriosclerosis in populations with prediabetes and new-onset diabetes. As a basic characteristic of type 2 diabetes mellitus (T2DM), insulin resistance plays an important role in the development and progression of arteriosclerosis. Insulin resistance is present in prediabetes, and populations with prediabetes have a high risk of cardiovascular diseases. Therefore, early evaluation of early arteriosclerosis in patients with newonset diabetes and prediabetes is crucial for the prevention of cardiovascular and cerebrovascular diseases. In the present study, the baPWV, ABI, and FGF21 were used to evaluate early arteriosclerosis in order to research the specific risk factors.

\section{Materials and methods}

\section{Study population}

This was a retrospective study. The participants included in this study were screened for diabetes in the endocrinology department of our hospital from January 2019 to July 2019. After undergoing an oral glucose tolerance test (OGTT), 48 participants had normal glucose tolerance (NGT), 34 had impaired glucose regulation (also known as prediabetes), and 66 had new-onset T2DM. Diabetes mellitus is a group of metabolic diseases characterized by hyperglycemia. High blood sugar is caused by a defective secretion of insulin, impaired biological function, or both. Long-term hyperglycemia leads to the chronic damage and dysfunction of various tissues, especially the eyes, kidneys, heart, blood vessels, and nerves. The development of diabetes is divided into three stages; the first stage is called the 'high-risk group', the second stage is called 'prediabetes' and the third stage is called 'diabetes'. Therefore, failing to meet the diagnostic criteria for diabetes is called prediabetes. This study was approved by the Ethics Committee of the First Hospital of Qinhuangdao (batch number: 2018H010), and informed consent was obtained from all the participants.

\section{Inclusion and exclusion criteria}

Inclusion criteria: (i) patients' age was older than 18 years old and (ii) patients signed their informed consent. Exclusion criteria: (i) patients had acute glucose metabolic disorder; (ii) patients had acute infection; (iii) patients had liver and renal dysfunction; (iv) patients had acute cardiovascular disease and (v) patients had cerebrovascular diseases.

\section{Physical measurement and laboratory test}

Height, weight, systolic blood pressure (SBP), and diastolic blood pressure (DBP) were measured by trained staff, and the BMI $\left(\mathrm{kg} / \mathrm{m}^{2}\right)$ was calculated. Fasting venous blood from the eligible participants was drawn the next morning after they were water fasted for $8 \mathrm{~h}$. A fully automatic biochemical analyzer (Hitachi) was used to detect the total cholesterol (TC), triglyceride, high-density lipoprotein cholesterol, and low-density lipoprotein cholesterol (LDL-C) levels. For the OGTT, $75 \mathrm{~g}$ of anhydrous glucose was taken orally under the fasting state. It was dissolved in $250-300 \mathrm{~mL}$ of water and consumed within 5-10 min. The venous blood was

This work is licensed under a Creative Commons Attribution-NonCommercial-NoDerivatives 4.0 elfternationab ficense.ifica.com at 04/26/2023 01:23:13PM 
separately collected under the fasting state $2 \mathrm{~h}$ after the administration of the glucose. The fasting plasma glucose (FPG), 2-h plasma glucose (2hPG), fasting insulin (FINS), and 2 -h insulin (2hINS) levels were detected. In addition, $4 \mathrm{~mL}$ of blood was drawn under the fasting state. The serum was separated and stored in a refrigerator at $-80^{\circ} \mathrm{C}$ for testing. An ELISA was used for detecting the FGF21. The kit was provided by BIM Company (USA), and the detection was performed according to the manufacturer's instructions. The serum insulin level was detected using a chemiluminescence microparticle immunoassay. The kit was provided by Abbott Laboratories. The homeostasis model was used to assess the insulin resistance index $($ HOMA-IR $=$ FPG $\times$ FINS/22.5).

\section{Measurement of baPWV and $A B I$ and the definition of abnormalities}

An arteriosclerosis tester (model: HBP-8000) produced by Omron Corporation (Japan) was used. The baPWV and ABI were measured by specially trained technicians. The room temperature of the examination room was maintained at $22-25^{\circ} \mathrm{C}$. Before the measurement, the participants were told not to smoke and to remain in a resting state for $>5$ min. They were also told to wear thin clothes and stay quiet and keep their entire body relaxed at the beginning of the measurement. Subsequently, the participants were placed in a horizontal position without a pillow. The palms of both hands were upward and placed by the sides of the body. Blood pressure cuffs were bound to the upper arms and the ankles of the lower limbs. The cuff balloon mark on the upper arm was aligned with the brachial artery, and the lower edge of the cuff was $2-3 \mathrm{~cm}$ from the cross-striation of the cubital fossa. The cuff balloon mark on the lower limb was located on the medial lower limb, and the lower edge of the cuff was $1-2 \mathrm{~cm}$ from the medial malleolus. The measurements were taken twice by the device, and the second measurements were the final results. The average values of the baPWV and ABI on the left and right sides were calculated. The assessment criteria developed by the American College of Cardiology (1993) were used: A baPWV of $<1400 \mathrm{~cm} / \mathrm{s}$ indicated normal arterial stiffness, and a baPWV of $>1400 \mathrm{~cm} / \mathrm{s}$ indicated arteriosclerosis. The diagnostic criteria proposed by the American Heart Association/American College of Cardiology were used: an $\mathrm{ABI}$ of $<0.9$ was defined as peripheral vascular disease.

\section{Sample size estimation}

The sample size was calculated by pass 12 software.

\section{Statistical analysis}

We used the software program SPSS 13.0 (IBM) to conduct the statistical analysis. The continuous variables of normal distribution were expressed as the mean \pm S.D., the continuous variables of non-normal distribution were expressed as the median (interquartile range), and the categorical variables were expressed as the frequency (percentage (\%)). For multiple comparisons, each value was compared by one-way ANOVA followed by Dunnett's test when each datum was conformed to the normal distribution, while the non-normally distributed continuous data were compared using non-parametric tests. The counting data were tested by the chi-square test. The correlation was analyzed using the Pearson correlation. The risk factors were screened by multiple linear regression analysis. The ABI and baPWV were used as the dependent variables, and age, BMI, SBP, DBP, FPG, 2hPG, FINS, 2hINS, TC, LDL-C, HOMA-IR, and FGF-21 were used as the independent variables. A $P$-value of $<0.05$ was considered statistically significant.

\section{Results}

\section{Results of analysis of general clinical indicators among the three groups}

The ANOVA showed statistically significant differences among the three groups in age, SBP, FPG, 2hPG, 2hINS, and HOMA-IR, all of which showed an increasing trend, except 2hINS. The age and $2 \mathrm{hPG}$ were higher in the new-onset diabetes group than in the other two groups and were higher in the prediabetes group than in the NGT group; the differences were statistically significant $(P<0.05)$. The SBP and FPG were higher in the prediabetes and new-onset diabetes groups than in the NGT group, but no statistically significant difference was found between the prediabetes and new-onset diabetes groups. The $2 \mathrm{hINS}$ was higher in the prediabetes group than in the NGT and new-onset diabetes groups, but no statistically significant difference was observed between the latter two groups. The HOMA-IR was higher in the new-onset diabetes group than in the other two groups, and the differences were statistically significant $(P<0.05)$, as shown in Table 1.

\section{Results for comparison of FGF21, baPWV, and ABI among the three groups}

The ANOVA showed statistically significant differences among the three groups in the FGF21 and baPWV $(P<0.05)$,

This work is licensed under a Creative Commons Attribution-NonCommercial-NoDerivatives 4.0 Internationad ticense.ifica.com at 04/26/2023 01:23:13PM 
Table 1 Comparison of clinical data and biochemical indicators among the three groups.

\begin{tabular}{l}
\hline Group \\
\hline Age $($ years $)$ \\
BMI $\left(\mathrm{kg} / \mathrm{m}^{2}\right)$ \\
SBP $(\mathrm{mmHg})$ \\
DBP $(\mathrm{mmHg})$ \\
TG $(\mathrm{mmol} / \mathrm{L})$ \\
TC $(\mathrm{mmol} / \mathrm{L})$ \\
HDL-c $(\mathrm{mmol} / \mathrm{L})$ \\
LDL-c $(\mathrm{mmol} / \mathrm{L})$ \\
FPG $(\mathrm{mmol} / \mathrm{L})$ \\
2hPG $(\mathrm{mmol} / \mathrm{L})$ \\
FINS $(\mu U / \mathrm{mL})$ \\
$2 \mathrm{hINS}(\mu \mathrm{U} / \mathrm{mL})$ \\
HOMA-IR \\
FGF21 \\
ABI \\
BaPWV $(\mathrm{m} / \mathrm{s})$ \\
Smoking rate $(\%)$
\end{tabular}

\begin{tabular}{c}
\hline NGT group $(n=48)$ \\
\hline $47.85 \pm 9.344$ \\
$25.58 \pm 3.26$ \\
$119.71 \pm 14.89$ \\
$77.85 \pm 10.83$ \\
$1.79 \pm 1.05$ \\
$5.34 \pm 1.13$ \\
$1.48 \pm 0.36$ \\
$2.81 \pm 0.887$ \\
$5.03 \pm 0.50$ \\
$6.20 \pm 1.12$ \\
$8.08 \pm 4.43$ \\
$32.18 \pm 21.12$ \\
$1.90 \pm 0.81$ \\
$60.23 \pm 14.84$ \\
$1.18 \pm 0.10$ \\
$1366.54 \pm 216.53$ \\
$22.9 \%(11)$
\end{tabular}

\begin{tabular}{c} 
Prediabetic group $(n=34)$ \\
\hline $52.58 \pm 7.71^{\#}$ \\
$27.00 \pm 2.73$ \\
$128.15 \pm 15.77^{\#}$ \\
$81.01 \pm 8.99$ \\
$1.79 \pm 0.98$ \\
$5.05 \pm 0.94$ \\
$1.37 \pm 0.35$ \\
$2.68 \pm 0.71$ \\
$5.49 \pm 0.71^{\#}$ \\
$9.24 \pm 1.13^{\#}$ \\
$8.91 \pm 4.56$ \\
$67.00 \pm 57.71^{\#}$ \\
$2.31 \pm 0.79$ \\
$70.78 \pm 12.82$ \\
$1.20 \pm 0.09$ \\
$1469.09 \pm 167.30^{\#}$ \\
$23.5 \%(8)$
\end{tabular}

\begin{tabular}{c}
\hline New-onset diabetic group $(n=66)$ \\
\hline $58.32 \pm 7.77^{\#, \&}$ \\
$26.04 \pm 3.14$ \\
$129.76 \pm 15.23^{\#}$ \\
$80.31 \pm 11.38$ \\
$2.03 \pm 1.61$ \\
$5.49 \pm 1.40$ \\
$1.34 \pm 0.29$ \\
$2.91 \pm 0.96$ \\
$7.36 \pm 2.53^{\#}$ \\
$13.59 \pm 4.32^{\#, \&}$ \\
$9.30 \pm 10.36$ \\
$38.41 \pm 30.28^{\&}$ \\
$3.09 \pm 2.22^{\#, \&}$ \\
$78.20 \pm 20.60^{\#}$ \\
$1.18 \pm 0.08$ \\
$1611.92 \pm 297.10^{\# \&}$ \\
$21.2 \%(14)$
\end{tabular}

$\begin{array}{r}\hline \multicolumn{1}{c}{\boldsymbol{F}} \\ \hline 22.447 \\ 2.132 \\ 6.425 \\ 1.099 \\ 0.634 \\ 1.438 \\ 2.416 \\ 0.816 \\ 27.706 \\ 85.841 \\ 0.351 \\ 9.915 \\ 8.112 \\ 8.803 \\ 0.896 \\ 14.020 \\ 0.954 \\ \hline\end{array}$

$\begin{array}{r}\multicolumn{1}{c}{\boldsymbol{P}} \\ \hline<0.001 \\ 0.122 \\ 0.002 \\ 0.333 \\ 0.532 \\ 0.241 \\ 0.093 \\ 0.444 \\ <0.001 \\ <0.001 \\ 0.704 \\ <0.001 \\ <0.001 \\ <0.001 \\ 0.410 \\ <0.001 \\ 0.539 \\ \hline\end{array}$

$\# P<0.05$, compared with the NGT group; ${ }^{\&} P<0.05$, compared with the IGT group. 2hINS, 2-h insulin; 2hPG, 2-h plasma glucose; ABI, ankle-brachial index; baPWV, brachial-ankle pulse wave velocity; DBP, diastolic blood pressure; FGF21, Fibroblast growth factor 21; FINS, fasting insulin; FPG, fasting plasma glucose; HDL-c, high-density lipoprotein cholesterol; HOMA-IR, the insulin resistance index was assessed using the homeostasis model; LDL-c, low-density lipoprotein cholesterol; SBP, systolic blood pressure; TC, total cholesterol; TG, triglyceride.

which showed an increasing trend. The baPWV was higher in the new-onset diabetes group than in the other two groups and higher in the prediabetes group than in the NGT group; the differences were statistically significant $(P<0.05)$. The FGF21 was higher in the new-onset diabetes group than in the NGT group $(P<0.05)$, but no statistically significant differences were found between the prediabetes and new-onset diabetes groups. Furthermore, no statistically significant differences were observed in the ABI among the three groups, as shown in Table 1.

\section{Results of the pearson correlation analysis of baPWV}

Age, SBP, DBP, FPG, 2hPG, and FGF21 were positively correlated with the baPWV, as shown in Table 2.

\section{Results of the multiple linear regression analysis of the risk factors for baPWV}

The baPWV was used as the dependent variable, and age, BMI, SBP, DBP, TC, LDL-C, HOMA-IR, and FGF-21 were used as independent variables to perform the multiple linear regression analysis. The results revealed that SBP, FGF21, and HOMA-IR were independent risk factors for baPWV, as shown in Table 3. When the baPWV was used as the dependent variable, and age, BMI, SBP, DBP, FPG, 2hPG, FINS, 2hINS, TC, LDL-C, and FGF-21 were used as the independent variables to perform the multiple linear regression analysis, the results revealed that FPG, SBP, and FGF21 were independent risk factors for baPWV, as shown in Table 4.

\section{Discussion}

The results of the study showed statistically significant differences between the new-onset T2DM and prediabetes groups and the NGT group in age, SBP, FPG, 2hPG, 2hINS,

Table 2 Correlation of BaPWV and FGF21 with other variables.

\begin{tabular}{|c|c|c|c|c|}
\hline \multirow[b]{2}{*}{ Variables } & \multicolumn{2}{|c|}{ BaPWV } & \multicolumn{2}{|c|}{ FGF21 } \\
\hline & $r$ & $P$ & $r$ & $P$ \\
\hline Age (years) & 0.469 & $<0.001$ & 0.271 & 0.001 \\
\hline $\mathrm{BMI}\left(\mathrm{kg} / \mathrm{m}^{2}\right)$ & -0.122 & 0.141 & -0.048 & 0.566 \\
\hline SBP (mmHg) & 0.356 & $<0.001$ & 0.127 & 0.123 \\
\hline DBP (mmHg) & 0.235 & 0.004 & 0.041 & 0.617 \\
\hline TG (mmol/L) & 0.036 & 0.666 & 0.084 & 0.310 \\
\hline TC (mmol/L) & 0.147 & 0.076 & 0.022 & 0.791 \\
\hline $\begin{array}{l}\text { HDL-C } \\
(\mathrm{mmol} / \mathrm{L})\end{array}$ & 0.047 & 0.571 & -0.137 & 0.098 \\
\hline LDL-c (mmol/L) & 0.132 & 0.111 & 0.037 & 0.655 \\
\hline FPG (mmol/L) & 0.468 & $<0.001$ & 0.170 & 0.038 \\
\hline 2hPG (mmol/L) & 0.512 & $<0.001$ & 0.351 & 0.000 \\
\hline FINS $(\mu \mathrm{U} / \mathrm{mL})$ & -0.066 & 0.430 & 0.050 & 0.550 \\
\hline 2hINS ( $\mu U / m L)$ & -0.137 & 0.105 & -0.059 & 0.487 \\
\hline HOMA-IR & 0.370 & $<0.001$ & 0.068 & 0.416 \\
\hline FGF21 & 0.429 & $<0.001$ & - & - \\
\hline
\end{tabular}

This work is licensed under a Creative Commons Attribution-NonCommercial-NoDerivatives 4.0 elnternationad dicense.ifica . com at $04 / 26 / 2023$ 01:23:13PM 
Table 3 Regression analysis results of the risk factors for BaPWV.

\begin{tabular}{l}
\hline Variable \\
\hline Constant \\
SBP \\
FGF-21 \\
HOMA-IR \\
\hline
\end{tabular}

$\begin{array}{r}\hline \multicolumn{1}{c}{\text { B }} \\ \hline 293.979 \\ 4.832 \\ 4.187 \\ 47.571 \\ \hline\end{array}$

$\begin{array}{r}\hline \multicolumn{1}{c}{\text { s.E. }} \\ \hline 178.015 \\ 1.694 \\ 0.843 \\ 11.018 \\ \hline\end{array}$

\begin{tabular}{c}
\hline Beta \\
\hline- \\
0.282 \\
0.341 \\
0.297 \\
\hline
\end{tabular}

\begin{tabular}{c}
\hline $\mathbf{t}$ \\
\hline 1.651 \\
2.853 \\
4.969 \\
4.318 \\
\hline
\end{tabular}

\begin{tabular}{c}
\hline $\boldsymbol{P}$ \\
\hline 0.101 \\
0.005 \\
0.000 \\
0.000 \\
\hline
\end{tabular}

\begin{tabular}{c}
\hline $\mathbf{9 5 \%} \mathbf{~ C l}$ \\
\hline$-57.966 \sim 645.923$ \\
$1.484 \sim 8.181$ \\
$2.521 \sim 5.852$ \\
$25.788 \sim 69.354$ \\
\hline
\end{tabular}

Age, BMI, SBP, DBP, TC, LDL-C, HOMA-IR, and FGF-21 were used as the independent variables.

and HOMA-IR. Compared with the patients who did not have diabetes, the patients with T2DM had double the risk of developing cardiovascular diseases (11). The FPG level increased by $1 \mathrm{mmol} / \mathrm{L}(18 \mathrm{mg} / \mathrm{dL})$, and the risk of a cardiovascular event or death increased by $17 \%$ (12). There are some common risk factors for diabetic vascular change and arteriosclerosis, which is caused by other reasons, such as aging, gender, obesity, hyperlipidemia, and hypertension. Diabetes is pathophysiologically characterized by insulin resistance, hyposecretion, and hyperglycemia; diabetic vascular changes are also closely related to it.

However, in this study, the 2hINS showed an increasing trend, suggesting that the older the age, the higher the incidence of diabetes. The insulin resistance of the patients with new-onset diabetes was higher than that of the patients with prediabetes. The 2hINS was the highest in the patients with prediabetes, indicating that the delayed insulin secretion in patients with prediabetes might be more significant than that in patients with diabetes.

This study used the recognized noninvasive indicators of baPWV and ABI and the cell biological marker FGF21 to reflect early arteriosclerosis. The baPWV was higher in the new-onset diabetes group than in the prediabetes group and higher in the prediabetes group than in the NGT group. Statistically significant differences in FGF21 were observed among the three groups. It was higher in the new-onset diabetes group than in the NGT group, but no significant differences were noted between the prediabetes group and the other two groups. Moreover, no significant difference in the ABI was found among the three groups. Further correlation and regression analyses showed that in addition to the SBP, the fasting blood glucose and HOMA-IR were closely related to the baPWV. As a method for detecting arterial stiffness, the baPWV has been widely used in the early assessment and prediction of arteriosclerotic diseases. It is a specific and sensitive indicator reflecting vascular aging. Previous studies have confirmed a significant increase in the baPWV in patients with T2DM; however, there have been few studies on the baPWV in patients with prediabetes and new-onset diabetes. Some studies have reported the baPWV in patients with impaired fasting glucose (IFG). A study conducted by Ohnish et al. (13) on the Japanese population found an increase in the baPWV in populations with IFG compared with individuals with the ideal blood glucose level. Lukich et al. (14) analyzed the effect of IFG on carotid and radial arterial PWV in the Caucasus population and found an increase in the PWV in the IFG group compared with the group with the ideal blood glucose level.

In this study, the HOMA-IR and baPWV in the patients with prediabetes increased significantly, suggesting that diabetic vascular disease might occur in prediabetes. In addition to the progression of diabetes at a rate of $5-10 \%$ per year for the population with prediabetes, an abnormal glucose metabolism can increase the risk of macrovascular complications, such as coronary heart disease and stroke (11). It also increases the total mortality and cardiovascular disease mortality $(15,16)$. Patients with prediabetes have a significantly increased risk of macrovascular complications. The mechanism is related to hyperinsulinemia, insulin resistance, dyslipidemia, abnormal blood pressure, vascular endothelial dysfunction, oxidative stress, and inflammatory response in this population.

Insulin resistance is considered to be the common pathophysiological basis of metabolic disorders and

Table 4 Regression analysis results of the risk factors for BaPWV.

\begin{tabular}{lr}
\hline Variable & \multicolumn{1}{c}{ B } \\
\cline { 1 - 1 } Constant & 310.971 \\
SBP & 4.675 \\
FGF-21 & 3.029 \\
FPG & 40.651 \\
\hline
\end{tabular}

$\begin{array}{r}\hline \multicolumn{1}{c}{\text { S.E. }} \\ \hline 178.248 \\ 1.740 \\ 0.885 \\ 12.343 \\ \hline\end{array}$

\begin{tabular}{c}
\hline Beta \\
\hline- \\
0.273 \\
0.240 \\
0.290 \\
\hline
\end{tabular}

\begin{tabular}{c}
\hline $\mathbf{t}$ \\
\hline 1.745 \\
2.686 \\
3.421 \\
3.294
\end{tabular}

\begin{tabular}{c}
\hline $\boldsymbol{P}$ \\
\hline 0.083 \\
0.008 \\
0.001 \\
0.001 \\
\hline
\end{tabular}

\begin{tabular}{c}
$\mathbf{9 5 \%} \mathbf{C l}$ \\
\hline$-41.671 \sim 663.613$ \\
$1.232 \sim 8.118$ \\
$1.278 \sim 4.781$ \\
$16.233 \sim 65.070$
\end{tabular}

Age, BMI, SBP, DBP, FPG, 2hPG, FINS, 2hINS, TC, LDL-C, and FGF-21 were used as the independent variables.

https://ec.bioscientifica.com

https://doi.org/10.1530/EC-21-0202 (c) 2021 The authors Published by Bioscientifica Ltd

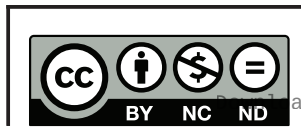

This work is licensed under a Creative Commons Attribution-NonCommercial-NoDerivatives 4.0 International dicense ifica.com at 04/26/2023 01:23:13PM 
cardiovascular diseases. It is also an important cause of atherosclerosis. Furthermore, atherosclerosis is the pathological basis for diabetic patients and nondiabetic patients, including those with multiple cardiovascular and cerebrovascular diseases, such as myocardial infarction and stroke. Animal experiments and clinical studies have demonstrated that vascular calcification and arteriosclerosis are associated with insulin resistance, which promotes vascular calcification $(17,18,19)$. The mechanism of insulin resistance is that the metabolic signaling pathway of IRS/PI3K/Akt is impaired, while the growth signaling pathway of Shc/Ras/MAPK is enhanced in a compensatory way. The metabolic signaling pathway has an anti-atherosclerotic effect, while the growth signaling pathway has an atherogenic effect. An impaired metabolic signaling pathway is the initial factor during insulin resistance and promotes the compensatory enhancement of the growth signaling pathway. Both have synergistic effects and comprise a vicious self-enhanced cycle process. This study showed that HOMA-IR was an independent risk factor for the baPWV.

Previous studies have shown that serum FGF21 levels are significantly elevated in patients with T2DM $(20,21)$. This study found that the FGF21 levels were elevated in patients with prediabetes and new-onset diabetes. Recent studies have revealed that FGF21 protects against lipotoxicity-induced pancreatic $\beta$-cell dysfunction by regulating AMP-activated protein kinase signal transduction and lipid metabolism (22). Therefore, it is considered that an increase in the FGF21 levels in diabetic patients is a compensatory increase. While FGF21 is preferentially produced in the liver, it has been identified as an endocrine and metabolic hormone due to its effects on lipids, glucose metabolism, insulin sensitivity, and energy balance (23). Studies have found that the serum FGF21 levels are significantly elevated in patients with carotid atherosclerotic plaques (24). Chow et al. (25) proposed that FGF21 is independent of the known cardiovascular risk factors and that its serum level is positively correlated with carotid atherosclerosis. In animal and in vitro studies, FGF21 has been shown to improve lipid distribution and inhibit key processes of the pathogenesis of atherosclerosis. It acts on the cardiovascular system through adiponectin-dependent and adiponectin-independent mechanisms (26). Yan et al. (8) found that FGF21 protects the cells from premature aging induced by $\mathrm{H}_{2} \mathrm{O}_{2}$ by delaying the replicative senescence of the endothelial cells. This study found that FGF21 was positively correlated with the baPWV and was an independent risk factor for it.
This study found no significant differences in the ABI levels among the three groups. This was probably due to the fact that the study population comprised of individuals screened for prediabetes and new-onset diabetes. The age range of the study population was 30-70 years.

It is estimated that $45.8 \%$ of patients with adult-onset diabetes (174.8 million) are undiagnosed worldwide, and the proportion ranges from 24.1 to $75.1 \%$ in different countries (27). Multiple studies have shown that upon combining the early detection of undiagnosed diabetes with effective prevention, such as health examinations or opportunistic screening methods, an early identification of abnormal glucose metabolism and timely interventions may be helpful to prevent and delay diabetic complications $(28,29)$.

There were several limitations in this study. First, this trial was not a randomized controlled trial. Secondly, this study was only a single-center trial. Thirdly, the sample size was limited, and, therefore, another trial with a large sample size should be conducted in the future.

\section{Conclusion}

Patients with prediabetes and new-onset diabetes may have more significant early arteriosclerosis. The blood glucose level and insulin resistance index may be the independent risk factors for early arteriosclerosis.

\section{Declaration of interest}

The authors declare that there is no conflict of interest that could be perceived as prejudicing the impartiality of the research reported.

\section{Funding}

This work did not receive any specific grant from any funding agency in the public, commercial, or not-for-profit sector.

\section{Ethical statement}

This study was approved by the ethics committee of the First Hospital of Qinhuangdao, and written informed consents were obtained from all participants. The study complied with the Declaration of Helsinki.

\section{Consent to publication}

All authors' gave final approval of the version to be published.

\section{Availability of data and materials}

The data that support the findings of this study are available from the ethics committee of the First Hospital of Qinhuangdao but restrictions apply to the availability of these data, which were used under license for the current study, and so are not publicly available. Data are, however, 
available from the authors upon reasonable request and with permission of the ethics committee of the First Hospital of Qinhuangdao.

\section{Author contribution statement}

Liu X L and Lu Q were involved in the conception and design of the research . Liu $X L$ and Liu $L X$ were involved in the acquisition of data. Wang $\mathrm{R}$ was involved in analysis and interpretation of the data. Jia $\mathrm{XJ}$ performed the statistical analysis. Liu X L, Liu B B, Ma N and Lu Q were involved in writing the manuscript. Liu $X L$ did the critical revision of the manuscript for intellectual content. None of the authors was involved in obtaining the finance.

\section{References}

1 Lanzer P, Boehm M, Sorribas V, Thiriet M, Janzen J, Zeller T, Hilaire CS \& Shanahan C. Medial vascular calcification revisited: review and perspectives. European Heart Journal 201435 1515-1525. (https://doi. org/10.1093/eurheartj/ehu163)

2 Li BY, Gao HQ, Li XL, Liu YP \& Wang M. Correlation between brachial ankle pulse wave velocity and arterial compliance and cardiovascular risk factors in elderly patients with arterioscler-osis. Hypertension Research 200629 309-314. (https://doi.org/10.1291/hypres.29.309)

3 Jia EZ, An FH, Liu P, Li F, Mao HW, Cui WJ \& Xu HY. Relationship between brachial-ankle pulse wave velocity and cardiovascular risk factors: a multi-ethnic study. Internal Medicine 201251 537-543. (https://doi.org/10.2169/internalmedicine.51.6480)

4 Sonoda H, Takase H, Dohi Y \& Kimura G. Factors associated with brachial-ankle pulse wave velocity in the general population. Journal of Human Hypertension 201226 701-705. (https://doi.org/10.1038/ jhh.2011.100)

5 Takase H, Dohi Y, Toriyama T, Okado T, Tanaka S, Sonoda H, Sato K \& Kimura G. Brachial-ankle pulse wave velocity predicts increase in blood pressure and onset of hypertension. American Journal of Hypertension 201124 667-673. (https://doi.org/10.1038/ajh.2011.19)

6 Kim YA, Kim ES, Hwang HK, Lee KB, Lee S, Jung JW, Kwon YJ, Cho DH, Park SS, Yoon J, et al. Prevalence and risk factors for the peripheral neuropathy in padents with peripheral arterial occlusive disease. Vascular Specialist International 201430 125-132. (https://doi. org/10.5758/vsi.2014.30.4.125)

7 Vu TH, Stamler J, Liu K, McDermott MM, Lloyd-Jones DM, Pirzada A, Garside DB \& Daviglus ML. Prospective relationship of low cardiovascular risk factor profile at younger ages to ankle-brachial index: 39-year follow-up - the Chicago Healthy Aging Study. Journal of the American Heart Association 20121 e001545. (https://doi.org/10.1161/ JAHA.112.001545)

8 Yan JH, Wang JL, Huang HJ, Huang Y, Mi T, Zhang CT \& Zhang L. Fibroblast growth factor 21 delayed endothelial replicative senescence and protected cells from $\mathrm{H} 2 \mathrm{O} 2$-induced premature senescence through SIRT1. American Journal of Translational Research 20179 4492-4501.

9 Qureshi G, Brown R, Salciccioli L, Qureshi M, Rizvi S, Farhan S \& Lazar J. Relationship between aortic atherosclerosis and non-invasive measures of arterial stiffness. Atherosclerosis 2007195 e190-e194. (https://doi.org/10.1016/j.atherosclerosis.2007.06.021)

10 Dang VT, Zhong LH, Huang A, Deng A \& Werstuck GH. Glycosphingolipids promote pro-atherogenic pathways in the pathogenesis of hyperglycemia-induced accelerated atherosclerosis. Metabolomics 201814 92. (https://doi.org/10.1007/s11306-018-1392-2)

11 Emerging Risk Factors Collaboration, Sarwar N, Gao P, Seshasai SR, Gobin R, Kaptoge S, Di Angelantonio E, Ingelsson E, Lawlor DA, Selvin E, et al. Diabetes mellitus, fasting blood glucose concentration, and risk of vascular disease: a collaborative meta-analysis of 102 prospective studies. Lancet 2010375 2215-2222. (https://doi. org/10.1016/S0140-6736(10)60484-9)

12 Anand SS, Dagenais GR, Mohan V, Diaz R, Probstfield J, Freeman R, Shaw J, Lanas F, Avezum A, Budaj A, EpiDREAM Investigators, et al. Glucose levels are associated with cardiovascular disease and death in an international cohort of normal glycaemic and dysglycaemic men and women: the EpiDREAM Cohort Study. European Journal of Preventive Cardiology 201219 755-764. (https://doi. org/10.1177/1741826711409327)

13 Ohnishi H, Saitoh S, Takagi S, Ohata JI, Isobe T, Kikuchi Y, Takeuchi H \& Shimamoto K. Pulse wave velocity as an indicator of atherosclerosis in impaired fasting glucose: the Tanno and Sobetsu Study. Diabetes Care 200326 437-440. (https://doi.org/10.2337/ diacare.26.2.437)

14 Lukich E, Matas Z, Boaz M \& Shargorodsky M. Increasing derangement of glucose homeostasis is associated with increased arterial stiffness in patients with diabetes, impaired fasting glucose and normal controls. Diabetes/Metabolism Research and Reviews 201026 365-370. (https:// doi.org/10.1002/dmrr.1086)

15 Stacey RB, Leaverton PE, Schocken DD, Peregoy JA \& Bertoni AG. Prediabetes the association with unrecognized myocardial infarction in the multi-ethnic study of atherosclerosis. American Heart Journal 2015170 923-928. (https://doi.org/10.1016/j.ahj.2015.08.003)

16 Magliano DJ, Söderberg S, Zimmet PZ, Cartensen B, Balkau B, Pauvaday V, Kowlessur S, Tuomilehto J, Alberti KGMM \& Shaw JE. Mortality, all-cause and cardiovascular disease, over 15 years in multiethnic mauritius: impact of diabetes and intermediate forms of glucose tolerance. Diabetes Care 201033 1983-1989. (https://doi. org/10.2337/dc10-0312)

17 Ong KL, McClelland RL, Rye KA, Cheung BMY, Post WS, Vaidya D, Criqui MH, Cushman M, Barter PJ \& Allison MA. The relationship between insulin resistance and vascular calcification in coronary arteries, and the thoracic and abdominal aorta: the Multi-Ethnic Study of Atherosclerosis. Atherosclerosis 2014236 257-262. (https:// doi.org/10.1016/j.atherosclerosis.2014.07.015)

18 Kou HJ, Deng J, Gao D, Song AQ, Han ZH, Wei J, Jin X, Ma R \& Zheng QS. Relationship among adiponectin, insulin resistance and atherosclerosis in non-diabetic hypertensive patients and healthy adults. Clinical and Experimental Hypertension 201840 656-663. (https://doi.org/10.1080/10641963.2018.1425414)

19 Nguyen N, Naik V \& Speer MY. Diabetes mellitus accelerates cartilaginous metaplasia and calcification in atherosclerotic vessels of LDLr mutant mice. Cardiovascular Pathology 201322 167-175. (https:// doi.org/10.1016/j.carpath.2012.06.007)

20 Eto K, Tumenbayar B, Nagashima SI, Tazoe F, Miyamoto M,Takahashi M, Ando A, Okada K, Yagyu H \& Ishibashi S. Distinct association of serum FGF21 or adiponectin levels with clinical parameters in patients with type 2 diabetes. Diabetes Research and Clinical Practice 201089 52-57. (https://doi.org/10.1016/j. diabres.2010.03.019)

21 Chen C, Cheung BMY, Tso AW, Wang YD, Law LSC, Ong KL, Wat NMS, Xu A \& Lam KSL. High plasma level of fibroblast growth factor 21 is an independent predictor of type 2 diabetes: a 5.4-year populationbased prospective study in Chinese subjects. Diabetes Care 201134 2113-2115. (https://doi.org/10.2337/dc11-0294)

22 Xie T, So WY, Li XY \& Leung PS. Fibroblast growth factor 21 protects against lipotoxicity-induced pancreatic $\beta$-cell dysfunction via regulation of AMPK signaling and lipid metabolism. Clinical Science 2019133 2029-2044. (https://doi.org/10.1042/CS20190093)

23 Kharitonenkov A, Shiyanova TL, Koester A, Ford AM, Micanovic R, Galbreath EJ, Sandusky GE, Hammond LJ, Moyers JS, Owens RA, et al. FGF-21 as a novel metabolic regulator. Journal of Clinical Investigation 2005115 1627-1635. (https://doi.org/10.1172/JCI23606)

24 An SY, Lee MS, Yi SA, Ha ES, Han SJ, Kim HJ, Kim DJ \& Lee KW. Serum fibroblast growth factor 21 was elevated in subjects with type 2 diabetes mellitus and was associated with the presence of carotid 
artery plaques. Diabetes Research and Clinical Practice 201296 196-203. (https://doi.org/10.1016/j.diabres.2012.01.004)

25 Chow WS, Xu A, Woo YC, Tso AWK, Cheung SCP, Fong CHY, Tse HF, Chau MT, Cheung BMY \& Lam KSL. Serum fibroblast growth factor-21 levels are associated with carotid atherosclerosis independent of established cardiovascular risk factors. Arteriosclerosis, Thrombosis, and Vascular Biology 201333 2454-2459. (https://doi.org/10.1161/ ATVBAHA.113.301599)

26 Kokkinos J, Tang S, Rye KA \& Ong KL. The role of fibroblast growth factor 21 in atherosclerosis. Atherosclerosis 2017257 259-265. (https:// doi.org/10.1016/j.atherosclerosis.2016.11.033)
27 Beagley J, Guariguata L, Weil C \& Motala AA. Global estimates of undiagnosed diabetes in adults. Diabetes Research and Clinical Practice 2014103 150-160. (https://doi.org/10.1016/j.diabres.2013.11.001)

28 Diabetes Prevention Program Research Group. The 10-year costeffectiveness of lifestyle intervention or metformin for diabetes prevention. An intent-to-treat analysis of the DPP/DPPOS. Diabetes Care 201235 723-730. (https://doi.org/10.2337/dc11-1468)

29 Siu AL \& US Preventive Services Task Force. Screening for abnormal blood glucose and type 2 diabetes mellitus: U.S. Preventive Services Task Force Recommendation Statement. Annals of Internal Medicine 2015163 861-868. (https://doi.org/10.7326/M15-2345)

Received in final form 26 April 2021

Accepted 6 May 2021

Accepted Manuscript published online 6 May 2021 (c) 2021 The authors Published by Bioscientifica Ltd
This work is licensed under a Creative Commons Attribution-NonCommercial-NoDerivatives 4.0 Internationab License ifica , com at $04 / 26 / 2023$ 01:23:13PM 